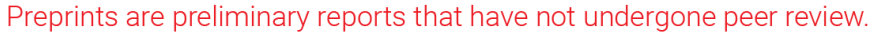 \\ They should not be considered conclusive, used to inform clinical practice, or referenced by the media as validated information. \\ Identification of Potential Hub Genes and Pathways Predicting Pathogenesis in Skin Cutaneous Melanoma
}

\section{Daishi Li}

Central South University

Yi Ge

Central South University

\section{Xinchen Ke}

Central South University

Jie Pan

Central South University

Yujie Wang

Central South University

Xuanlin Che

Central South University

Jia Guo

Central South University

Yi Wang

Central South University

Xianggui Wang

Central South University

Juan Su ( $\nabla$ sujuanderm@csu.edu.cn )

Central South University

\section{Xiang Chen}

Central South University

\section{Research Article}

Keywords: EXO1, Melanoma, DEGs, Rifabutin, bioinformatics

Posted Date: February 25th, 2022

DOI: https://doi.org/10.21203/rs.3.rs-1376326/v1 
License: (c) (i) This work is licensed under a Creative Commons Attribution 4.0 International License. Read Full License 


\section{Abstract}

Background: Malignant melanoma (MM) is one of the tumors with the worst prognosis among skin malignancies. However, its pathogenesis and potential therapeutic targets still need to be explored to a large extent.

Methods: In the present study, we found three gene expression profiles and screened differentially expressed genes (DEGs) between melanoma and normal tissues. The DEGs were dealt with gene ontology (GO), Kyoto encyclopedia of genes and genomes (KEGG), protein-protein interaction (PPI), and survival analyses, while the connectivity map (CMap) database was used to predict candidate small molecules that may reverse the biological state of MM

Results: we detected the expression of the most relevant core gene in vitro and checked the impact of the top predicted potential drug against the proliferation of MM cell lines. EXO1 were associated with unfavorable MM prognosis, ten hub genes were chose among the 77 up-regulated DEGs and 41 downregulated DEGs, and three promising small molecule drugs for treating MM were identified.

Simultaneously, we found that the highest predicted potential drug rifabutin significantly inhibits on the proliferation of melanoma cells and significantly reverses the expression of EX01.In summary, the findings of the present study suggest that the ten hub genes and pathways identified may be closely related to MM pathogenesis.

Conclusions: EX01 overexpression could be a hopeful biomarker for revealing poor prognosis in patients with $\mathrm{MM}$, while the rifabutin for the development of potential drugs to treat MM.

\section{Introduction}

Malignant melanoma (MM), a solid tumor produced by the malignant transformation of melanocytes from the skin and other organs, has an increasing incidence and a poor prognosis. Compared with other tumors originating from the epidermis and accessory organs, the cutaneous form of the disease is common in the Western world and is late onset with no obvious early symptoms, which causes the threequarters of the deaths related to skin cancer [1]. Despite various advances in surgery, chemotherapy, targeted therapy, and immunotherapy in recent decades, survival of patients with skin cutaneous melanoma (SKCM) is still limited[2]. Thus, there is a need to identify potential new biomarkers and the pathways and molecular mechanisms underlying carcinogenesis to develop novel diagnostic and treatment strategies for SKCM.

In recent years, with the rapid development of bioinformatics technology, bioinformatics has become increasingly popular for exploring the molecular mechanisms of diseases by data mining at the molecular level, which improved our understanding of cancer notably [3, 4]. The Gene Expression Omnibus (GEO) is an international public repository that collects data from different microarray platforms and provides a resource for data mining to uncover molecular variations in a wide variety of tumors, which is 
significant for clarifying the molecular mechanisms of tumor pathogenesis and discovering new potential biomarkers which could improve early diagnosis and prognosis[5].

In this study, in order to obtain melanoma hub genes, we screened DEGs between SKCM and normal tissues using bioinformatics analyses of GEO data and then performed Gene Ontology (GO) functional annotation analysis and Kyoto Encyclopedia of Genes and Genomes (KEGG) pathway enrichment analysis. Thereafter we identified ten hub genes related to SKCM by using protein-protein interaction (PPI) network analysis. To further verified our analysis's accuracy and reliability, we used Gene Expression Profiling Interactive Analysis (GEPIA) to verify their effects on survival. In addition, we identified potential small molecule drugs that could reverse SKCM-induced gene expression using connectivity map (CMap) database analysis. In the end, up-regulation of the most relevant core gene, EXO1, was validated in various SKCM cell lines. Meanwhile, we demonstrated that rifabutin, the top predicted drug, could inhibit SKCM cell proliferation and reverse EXO1 expression. Therefore, these potential small molecules were potential therapeutic candidates for SKCM to block EXO1 activity and future studies are essential to confirm their efficacy.

\section{Materials And Methods}

\section{Data source}

Gene expression data were downloaded from NCBI GEO databases (https://www.ncbi.nlm.nih.gov/geo/). Three gene expression profiles were selected: GSE12391, GSE15605, and GSE83583. The microarray data from GSE12391 was based on the GPL1708 Platform (Agilent-012391; Whole Human Genome Oligo Microarray), GSE15605 was based on the GPL570 Platform (HG-U133_Plus_2; Affymetrix Human Genome U133 Plus 2.0 Array), and GSE83583 was based on the GPL10558 Platform (Illumina HumanHT12 V4.0 expression beadchip).

\section{Identification of DEGs}

We used GEO2R online analysis tool (https://www.ncbi.nlm.nih.gov/geo/geo2r/) to identify DEGs between SKCM and normal or nevus samples. Fold-change (FC) and adjusted p-values were calculated, with a $|\log (F C)|$ in the top 2000 and an adj. P-value $<0.05$ defined as the cutoff criteria for DEGs. Overlapping DEGs were identified using the Venn diagram web tool (https://bioinformatics.psb.ugent.be/webtools/Venn/).

\section{Functional and pathway enrichment analysis}

Functional and pathway enrichment analysis $\mathrm{GO}$ provides a framework to describe molecular functions (MF), biological processes (BP), and cellular components (CC), while the KEGG database is widely used to elucidate biological pathways involving DEGs. Both GO annotation and KEGG pathway enrichment analyses were performed using Database for Annotation, Visualization and Integrated Discovery (DAVID) 
tools (https://david.ncifcrf.gov/). P-value $<0.05$ and gene counts $\geq 5$ were considered statistically significant.

\section{PPI network analysis and hub gene identification}

We used the STRING (http://stringdb.org/) online database to evaluate the potential PPIs among the DEGs identified. PPIs between DEG pairs with a combined score of $>0.400$ (medium confidence) were visualized as a network using Cytoscape software (www.cytoscape.org/), in which highly intra-connected nodes tend to be more important for maintaining the stability of the gene expression network. In addition, we used the Cytoscape CytoHubba plug-in to obtain the degree of each protein node and identify the top ten most connected hub genes.

\section{Hub gene expression and survival analysis}

The Cancer Genome Atlas (TCGA) data visualization web-tool, GEPIA2 (http://gepia2.cancerpku.cn/\#index), interactively analyzes cancer-related and normal genes [6]. In the present study, we used GEPIA2 for expression and survival analysis to evaluate the prognostic value of the identified hub genes in patients with SKCM. Hub genes significantly associated with survival rate (log rank $P$ value $<0.05)$ were considered to be core genes for SKCM prognosis.

\section{Screening of potential small molecule drugs}

CMap (https://portals.broadinstitute.org/cmap/) is a collection of genome-wide transcriptional expression data that enables the discovery of functional connections between drugs, genes, and diseases via transient changes in common gene expression [7]. We used Cmap to search for potential drugs which could reverse up-regulation of EX01, as well as the results were ranked according to their score. The 2D chemical structures of the top three small molecules were determined on the

\section{Cell lines and culture conditions}

Two human melanoma cell lines (A375 and SK-MEL-28) and one human keratinocyte (HaCaT) cells were purchased from the American Type Culture Collection (ATCC). All cells were grown in Dulbecco's modified Eagle's medium (BI) supplemented with $10 \%$ fetal bovine serum (ExCell) at $37^{\circ} \mathrm{C}$ in a $5 \% \mathrm{CO} 2$ humidified incubator.

\section{Western blot assay}

Cells were harvested in RIPA Lysis Buffer (DingGuo) with a protease inhibitor and phosphatase inhibitors (Selleck), and protein concentration was determined using a BCA assay kit (Beyotime). Protein samples $(35 \mu \mathrm{g})$ were subjected to $10 \%$ SDS-polyacrylamide gel electrophoresis (SDS-PAGE) and then transferred onto polyvinylidene fluoride membranes (Millipore). The membranes were blocked with $5 \%$ non-fat milk for one hour at room temperature and then incubated with following primary and secondary antibodies: EX01 (1:1000, Proteintech Group) and GAPDH (1:3000, Proteintech Group). Immunoreactive bands were 
visualized using an enhanced chemiluminescent kit (ECL+, NCM biotech) and measured using Quality One analysis software (BioRad).

\section{Cell proliferation assay}

Cells $\left(2 \times 10^{3}\right.$ per well) were seeded in a 96-well plate and allowed to adhere overnight in the culture medium containing $10 \%$ fetal bovine serum. Then, the cells were treated with various concentration of rifabutin (control, 10, 25, $50 \mu \mathrm{mol} / \mathrm{L}$ ) for 24,48 and 72 hours. The effect of spautin-1 or a combination of spautin-1 and cisplatin on cell viability was tested using the Nonradioactive Cell Proliferation Assay (3(4,5-dimethylthiazol-2-yl)5-(3-carboxymethoxyphenyl)-2-(4-sulfophenyl)-2H-tetrazolium, MTS) (Promega) according to the manufacturer's instructions. All assays were performed in triplicate.

\section{Results}

\section{DEG identification}

To identify DEGs in SKCM, we utilized three gene expression profiles: GSE12391, GSE15605, and GSE83583. GSE12391 included 56 melanoma tumor samples and 36 nevus samples. GSE15605 contained 58 melanoma samples and 16 marginal normal skin samples, while GSE83583 contained 81 SKCM samples and 3 melanocytes samples (Table 1). From these data, we obtained a total of 12000 DEGs, including 6000 up-regulated genes and 6000 down-regulated genes. In addition, the Venn diagram web-tool was used to intersect the three sets of DEGs and identify 118 that overlapped, of which 77 were significantly up-regulated and 41 were down-regulated (Fig. 1A, B).

Table 1

Sample composition of the three microarray datasets derived from the GEO database

\begin{tabular}{|llll|}
\hline Dataset ID & Melanoma samples & Normal or nevus samples & Total samples \\
\hline GSE12391 & 56 & 36 & 92 \\
\hline GSE15605 & 58 & 16 & 74 \\
\hline GSE83583 & 81 & 3 & 84 \\
\hline Abbreviations: GEO, Gene Expression Omnibus. \\
\hline
\end{tabular}

\section{DEG enrichment analysis}

We used DAVID to conduct GO functional and KEGG pathway enrichment analyses to elucidate the biological functions of the 118 DEGs identified. GO analysis indicated that the DEGs were mainly enriched in BPs including sister chromatid cohesion, chromosome segregation, mitotic nuclear division, DNA replication, viral process, regulation of signal transduction by p53 class mediator, cellular response to DNA damage stimulus, cell division, cell proliferation, negative regulation of transcription, DNA- 
templated, cell-cell adhesion, negative regulation of cell proliferation, inflammatory response, negative regulation of transcription from RNA polymerase II promoter, as well as CCs such as kinetochore, condensed chromosome kinetochore, chromosome, centromeric region, nucleus, cytosol, cytoplasm, spindle, nucleoplasm, nucleolus, membrane, proteinaceous extracellular matrix, centrosome, and in MFs containing protein binding, identical protein binding, enzyme binding, protein C-terminus binding, protein homodimerization activity, ATP binding, protein serine/threonine kinase activity, protein kinase activity, transcription factor binding, cadherin binding involved in cell-cell adhesion. In addition, KEGG pathway analysis indicated that the most enriched pathways were the pathways in cancer, cell cycle, hepatitis $\mathrm{C}$, Transcriptional misregulation in cancer, and proteoglycans in cancer (Table 2).(The table was placed at the end of the document text file.) 
Table 2

DEG functional and pathway enrichment analysis

\begin{tabular}{|c|c|c|c|c|}
\hline Category & Term & Description & Count & P-value \\
\hline BP & GO:0007062 & sister chromatid cohesion & 10 & 8.73E-10 \\
\hline BP & GO:0007059 & chromosome segregation & 6 & $1.39 \mathrm{E}-05$ \\
\hline BP & G0:0007067 & mitotic nuclear division & 9 & $1.60 \mathrm{E}-05$ \\
\hline BP & G0:0006260 & DNA replication & 6 & $6.85 \mathrm{E}-04$ \\
\hline BP & G0:0016032 & viral process & 7 & 0.002332771 \\
\hline BP & GO:1901796 & $\begin{array}{l}\text { regulation of signal transduction by p53 } \\
\text { class mediator }\end{array}$ & 5 & 0.002412016 \\
\hline BP & GO:0006974 & cellular response to DNA damage stimulus & 6 & 0.002518336 \\
\hline BP & GO:0051301 & cell division & 7 & 0.005046764 \\
\hline BP & G0:0008283 & cell proliferation & 7 & 0.006247998 \\
\hline BP & GO:0045892 & $\begin{array}{l}\text { negative regulation of transcription, DNA- } \\
\text { templated }\end{array}$ & 8 & 0.007334025 \\
\hline BP & G0:0098609 & cell-cell adhesion & 6 & 0.00767987 \\
\hline BP & GO:0008285 & negative regulation of cell proliferation & 7 & 0.009044158 \\
\hline BP & GO:0006954 & inflammatory response & 6 & 0.028591068 \\
\hline BP & G0:0000122 & $\begin{array}{l}\text { negative regulation of transcription from RNA } \\
\text { polymerase Il promoter }\end{array}$ & 8 & 0.044081553 \\
\hline $\mathrm{CC}$ & GO:0000776 & kinetochore & 8 & $6.44 \mathrm{E}-08$ \\
\hline $\mathrm{CC}$ & GO:0000777 & condensed chromosome kinetochore & 7 & $2.25 \mathrm{E}-06$ \\
\hline $\mathrm{CC}$ & G0:0000775 & chromosome, centromeric region & 6 & $5.03 E-06$ \\
\hline $\mathrm{CC}$ & GO:0005634 & nucleus & 43 & $1.63 \mathrm{E}-05$ \\
\hline $\mathrm{CC}$ & GO:0005829 & cytosol & 31 & $3.56 \mathrm{E}-05$ \\
\hline $\mathrm{CC}$ & GO:0005737 & cytoplasm & 38 & $6.10 \mathrm{E}-04$ \\
\hline $\mathrm{CC}$ & GO:0005819 & spindle & 5 & 0.001982375 \\
\hline $\mathrm{CC}$ & GO:0005654 & nucleoplasm & 23 & 0.00351478 \\
\hline $\mathrm{CC}$ & GO:0005730 & nucleolus & 10 & 0.012625274 \\
\hline $\mathrm{CC}$ & GO:0016020 & membrane & 17 & 0.028134024 \\
\hline
\end{tabular}




\begin{tabular}{|c|c|c|c|c|}
\hline Category & Term & Description & Count & P-value \\
\hline $\mathrm{CC}$ & G0:0005578 & proteinaceous extracellular matrix & 5 & 0.030305507 \\
\hline $\mathrm{CC}$ & GO:0005813 & centrosome & 6 & 0.039356285 \\
\hline MF & GO:0005515 & protein binding & 58 & 1.67E-05 \\
\hline MF & GO:0042802 & identical protein binding & 14 & $2.31 \mathrm{E}-05$ \\
\hline MF & GO:0019899 & enzyme binding & 8 & $6.86 \mathrm{E}-04$ \\
\hline MF & GO:0008022 & protein C-terminus binding & 6 & 0.001293246 \\
\hline MF & GO:0042803 & protein homodimerization activity & 10 & 0.00492987 \\
\hline MF & GO:0005524 & ATP binding & 15 & 0.005647052 \\
\hline MF & GO:0004674 & protein serine/threonine kinase activity & 7 & 0.006495727 \\
\hline MF & GO:0004672 & protein kinase activity & 6 & 0.021721961 \\
\hline MF & G0:0008134 & transcription factor binding & 5 & 0.037616963 \\
\hline MF & GO:0098641 & $\begin{array}{l}\text { cadherin binding involved in cell-cell } \\
\text { adhesion }\end{array}$ & 5 & 0.040122285 \\
\hline $\begin{array}{l}\text { KEGG } \\
\text { pathway }\end{array}$ & hsa05200 & Pathways in cancer & 10 & 2.19E-04 \\
\hline $\begin{array}{l}\text { KEGG } \\
\text { pathway }\end{array}$ & hsa04110 & Cell cycle & 5 & 0.004623304 \\
\hline $\begin{array}{l}\text { KEGG } \\
\text { pathway }\end{array}$ & hsa05160 & Hepatitis C & 5 & 0.005925678 \\
\hline $\begin{array}{l}\text { KEGG } \\
\text { pathway }\end{array}$ & hsa05202 & Transcriptional misregulation in cancer & 5 & 0.01300871 \\
\hline $\begin{array}{l}\text { KEGG } \\
\text { pathway }\end{array}$ & hsa05205 & Proteoglycans in cancer & 5 & 0.023656161 \\
\hline \multicolumn{5}{|c|}{$\begin{array}{l}\text { Abbreviations: DEG, differentially expressed gene; BP, biological process; CC, cellular component; } \\
\text { KEGG, Kyoto Encyclopedia of Genes and Genomes; GO, Gene Ontology. }\end{array}$} \\
\hline
\end{tabular}

\section{PPI network construction and hub gene identification}

Next, we analyzed PPIs between overlapping DEGs using STRING, identifying a network of relevant PPIs consisting of 116 nodes and 315 edges that were visualized using Cytoscape (Fig. 2A, B). Based on their degree of connectivity within the PPI network, the top ten hub genes were selected: EXO1, TOP2A, BUB1, RRM2, KIF23, TTK, DLGAP5, MCM10, PBK, NUF2 (Table 3), all of which were up-regulated in GEO. GEPIA 
of hub gene expression in SKCM revealed that the expression of all ten hub genes was significantly increased in 461 SKCM tissues compared with that in normal control tissues $(P<0.05$, Fig. 3$)$.

Table 3

Top ten hub genes with higher degree of connectivity

\begin{tabular}{|llll|}
\hline Gene symbol & Gene description & Degree & Regulation \\
\hline TOP2A & DNA topoisomerase 2-alpha & 42 & Up \\
\hline BUB1 & Mitotic checkpoint serine/threonine-protein kinase BUB1 & 42 & Up \\
\hline RRM2 & Ribonucleoside-diphosphate reductase subunit M2 & 42 & Up \\
\hline KIF23 & Kinesin-like protein KIF23 & 42 & Up \\
\hline EX01 & Exonuclease 1 & 42 & Up \\
\hline TTK & Dual specificity protein kinase TTK & 40 & Up \\
\hline DLGAP5 & Disks large-associated protein 5 & 40 & Up \\
\hline MCM10 & Protein MCM10 homolog & 40 & Up \\
\hline PBK & Phosphorylates MAP kinase p38 & 40 & Up \\
\hline NUF2 & Kinetochore protein Nuf2 & 40 & Up \\
\hline
\end{tabular}

Table 4

Three most significant small molecule drugs

\begin{tabular}{|llll|}
\hline Name & Score & Type & Description \\
\hline Rifabutin & 98.91 & conpound & $\begin{array}{l}\text { DNA directed DNA polymerase inhibitor, DNA directed RNA } \\
\text { polymerase inhibitor, protein synthesis inhibitor }\end{array}$ \\
\hline Otenzepad & 98.00 & conpound & acetylcholine receptor antagonist \\
\hline Mocetinostat & 96.69 & conpound & HDAC inhibitor, cell cycle inhibitor \\
\hline
\end{tabular}

\section{Hub gene survival analysis}

To investigate the prognostic value of the ten hub genes in SKCM, we used GEPIA2. High EXO1 and MCM10 expression were associated with unfavorable relapse-free survival in patients with SKCM $(\mathrm{P}<$ 0.05 , Fig. 4). Equally, patients with high expression of the other eight hub genes also had a tendency toward poor survival compared with that in patients showing low gene expression; however, this correlation was not significant ( $P>0.05$, Fig. 4). Therefore, EXO1 and MCM10 were identified as adverse prognostic factors in patients with SKCM. 


\section{In vitro validation of EX01 overexpression in SKCM cell lines}

To validate EXO1 as an overexpressed core gene in vitro, we investigated its expression levels in the two SKCM cell lines and the normal HaCaT cell line. Interestingly, higher EXO1 protein expression was detected in the SKCM cell lines (Fig. 5A, B), consistent with our observation that EX01 expression is significantly increased in SKCM compared with that in normal control tissues.

\section{Screening of potential small molecule drugs}

To predict candidate small molecules for treating SKCM, we used the CMap database. The chemical structures of the top three small molecules (rifabutin, otenzepad, and mocetinostat) are shown in Fig. 6. These small molecules were the most likely to reverse SKCM-related gene expression and could help to develop new targeted drugs to treat SKCM.

\section{Correlation between the inhibition of SKCM cell proliferation by rifabutin and EX01 expression}

To determine the value of these potential small molecule drugs for treating SKCM, we examined the effect of the top predicted potential drug, rifabutin, on the proliferation of SKCM cell lines. We found that the proliferation of two SKCM cell lines, as measured by a CCK-8 assay, was markedly inhibited after $24 \mathrm{~h}$ of exposure to rifabutin (Fig. 7A). Next, we explored whether treatment with rifabutin could reverse gene expression in SKCM by measuring EX01 protein expression using Western blot assays. Rifabutin distinctively inhibited the expression of EXO1, which is overexpressed in SKCM (Fig. 7B), suggesting that the inhibitory effect of rifabutin against SKCM cell proliferation could be related to its inhibition of EXO1 overexpression.

\section{Discussion}

SKCM is the most malignant tumor among all skin tumors, owing to its highly invasive and metastatic potential[8], and the prognosis is extremely poor. Although SKCM only accounts for $4 \%$ of all skin cancers, it accounts for nearly $80 \%$ of all skin cancer-related deaths[9-11]. Early melanoma can be effectively treated by prompt surgical resection [12]. For patients with unresectable melanoma or tumors that have developed distant metastases (unresectable stage III or IV or advanced melanoma), with the rise of immunotherapy and targeted therapy, such as immune checkpoint suppression agents, especially anti-CTLA-4 and anti-PD-1 antibodies can control the tumors of part of patients with advanced melanoma, and the prognosis can be significantly improved [13].However, the significant therapeutic response to immunotherapy is limited to a small number of patients and indications $[14,15]$.Additionally, the low response rate and drug tolerance make the treatment of malignant melanoma still challenging. Therefore, it is important to determine a more effective treatment target for malignant melanoma.

Based on the SKCM gene expression profile data in the GEO database, we identified 77 up-regulated DEGs and 41 down-regulated DEGs. These genes were connected with GOBP terms such as sister chromatid cohesion chromosome segregation, mitotic nuclear division (nuclear mitosis), DNA replication, 
regulation of signal transduction by p53 class mediator, cellular response to DNA damage stimulus and KEGG terms such as pathways in cancer, cell cycle, transcriptional misregulation in cancer, proteoglycans in cancer. To certain the kinship between different genes, we constructed a PPI network and identified 10 pivot genes: TOP2A, BUB1, RRM2, KIF23, EX01, TTK, DLGAP5, MCM10, PBK, NUF2. These genes are all up-regulated in melanoma, and based on GEPIA analysis, they are highly expressed in melanoma patients. Among them, we found that EX01 overexpression is a significant unfavorable prognostic factor for Melanoma patients $(P=0.00083)$, and we verified that EXO1 overexpression in melanoma cells in vitro. Simultaneously, we found that the highest predicted potential drug rifabutin significantly inhibits on the proliferation of melanoma cells and significantly reverses the expression of EXO1.

EXO1 is a member of the Rad2 nuclease family, with 5' to 3' dsDNA exonuclease and 5-Flap endonuclease activities, and plays a role in many important cell pathways[16], including DNA mismatch repair[17] homologous recombination [18], DNA replication [19], cell cycle checkpoint activation [20]. The $\mathrm{N}$-terminal and C-terminal of EXO1 can interact with mismatch repair proteins MSH3, MSH1, MLH1 to complete DNA mismatch repair[21].EXO1 is a key enzyme in homologous recombination (HR). Homologous recombination is mainly initiated by DNA double-strand breaks or single-strand gaps in double-stranded DNA[16].EXO1 is involved in the key process of repairing double-stranded DNA breaks in the $S$ and $G 2$ phases of the cell cycle. It regulates double-strand break repair and damage signaling decision-making by cutting the ends of human DNA[22]. At the same time, EX01 is an essential enzyme in the replication process [23]. In the S phase, EXO1 co-localizes with mismatch repair proteins $\mathrm{MSH} 2$ and cell cycle regulator PCNA [24], And through the PCNA interacting protein (PIPbox) motif located in the Cterminal region to physically interact with PCNA, thereby regulating the cell cycle[19, 25].

In addition, our enrichment pathway analysis shows that KEGG-related pathways such as pathways in cancer, transcriptional misregulation in cancer, proteoglycans in cancer. All of them indicate that EXO1 may be a key factor involved in the occurrence, development and evolution of cancer. Previous reports in the literature indicate that EXO1 has been proven to contribute to various tumors, including breast cancer [26],hepatocellular carcinoma [27, 28],prostate cancer [29],bladder Cancer [30],lung adenocarcinoma [31],cervical cancer [32].Besides, some of these studies has shown that the high expression of EX01 may be related to the poor prognosis of these malignant tumors. Lina Qi et al. found that EXO1 was significantly related to the prognosis of breast cancer [26] [33]. EX01 overexpression is tremendously associated with poor prostate cancer survival outcomes [29]. EXO1 is a potential prognostic biomarker in lung adenocarcinoma. It not only affects the prognosis, but is also related to the level of immune cell infiltration in the tumor microenvironment. EXO1 overexpression can reduce the infiltration of $\mathrm{B}$ cells and CD4 T cells in the lesion [31]. Meanwhile, Dongyun He et al. found that knocking down EXO1 can remarkably improve the doxorubicin resistance of ovarian cancer[34].

Rifabutin is a kind of rifamycin, which can specifically bind to the subunit of RNA polymerase of several bacteria. It also shows little or no activity to human RNA polymerase as a transcription inhibitor and can inhibit most bacteria [35-37]. Therefore, rifabutin has a broad spectrum of biological activities, effectively fighting group A streptococci staphylococci, Neisseriagonorrhoeae, Neisseria meningitidis, 
Haemophilus injluenzae, Haemophilus ducreyi, Campylobacter jejuni, Chlamydia trachomatis, and Toxoplasma gondii [38]. Because there are fewer drug interactions with AIDS treatment-related drugs, it is used to treat AIDS patients with tuberculosis and Mycobacterium avium complex [36]. Rifabutin can also be used in combination to eradicate Helicobacter pylori [39]. A prospective study suggests that rifabutin can be used for amoxicillin, clarithromycin, nitroimidazoles, fluoroquinolones, bismuth or tetracycline, etc., which is ineffective in the treatment of Helicobacter pylori infections [40]. Hidekazu Suzukid et al. believe that rifabutin can be used as a third-line or fourth-line rescue therapy to prevent gastritis caused by Helicobacter pylori infection and prolonged gastric cancer [41].At the same time, rifabutin can be used as a heat shock protein inhibitor to significantly inhibit the growth and proliferation of pancreatic cancer cells [42]. However, there are currently few studies on rifabutin as a heat shock protein inhibitor, and the specific mechanism and effect still need to be clarified. At present, some studies have shown that the mycobacterium tuberculosis(TB will be activated after immunotherapy [43].Existing studies have reported that cancer patients were infected with TB after receiving immune checkpoint blocking therapy, but due to the small number of samples, it is impossible to clarify the relationship between tuberculosis infection and immunotherapy [44].We found for the first time that rifabutin can inhibit the cell proliferation of melanoma cells as well as inhibit the expression of EX01. At the same time, it also provides better drug choices for patients who have been infected with tuberculosis after receiving immunotherapy. The specific mechanism still needs further research and exploration.

\section{Conclusion}

Above all, we used bioinformatics analysis to screen 118 DEGs between melanoma and normal tissues, and identified 10 key pivot genes and meaningful pathways that may play an essential role in the pathogenesis of melanoma. In particular,EXO1 overexpression is associated with poor prognosis of melanoma patients, so we speculate that it may be a new and promising biomarker for the survivals and prognosis of melanoma patients. In addition, in this study, we have proved that rifabutin may be a new and capable drug candidate for the treatment of melanoma, thus proposing a new treatment method for screening and developing potential anti-melanoma drugs.

\section{Abbreviations}

DEGs: Differentially expressed genes; MM: Melanoma; GEO: Gene Expression Omnibus; SKCM: skin cutaneous melanoma; GO: gene ontology; KEGG: Kyoto encyclopedia of genes and genomes; PPI protein-protein interaction; CMap: connectivity map.

\section{Declarations}

\section{Ethics approval and consent to participate}

Not applicable. 


\section{Consent for publication}

Not applicable.

\section{Availability of data and materials}

In this study, three gene expression profiles were downloaded from the GEO database under the accession number GSE12391, GSE15605, and GSE83583.

\section{Competing interests}

The authors declare that they have no competing interests.

\section{Funding}

This work was supported by the National Natural Science Foundation of China [grant numbers 82173009].

\section{Authors' contributions}

Daishi Li t,Yi Get have contributed equally to this work and shared the first authorship. The hypothesis and implementation plan were made by Yi Ge and Juan Su, Daishi Li and Yi Ge conducted the experiments and wrote the manuscript. Daishi Li was responsible for revising and submitting manuscript. The remaining members took part in the rest of the work. All authors contributed to the article and approved the submitted version.

\section{Acknowledgments}

At first, we must appreciate our dear tutor Juan Su for her support and encouragement as always. Alternatively, we would like to thank our team for providing us with valuable platform and resources.

\section{References}

1. Guo J, Zhang J, Liang L, Liu N, Qi M, Zhao S, et al. Potent USP10/13 antagonist spautin-1 suppresses melanoma growth via ROS-mediated DNA damage and exhibits synergy with cisplatin. J Cell Mol Med. 2020;24(7):4324-40.

2. Liu N, Qi M, Li K, Zeng W, Li J, Yin M, et al. CD147 regulates melanoma metastasis via the NFAT1MMP-9 pathway. Pigment Cell Melanoma Res. 2020;33(5):731-43.

3. Li MX, Jin LT, Wang TJ, Feng YJ, Pan CP, Zhao DM, et al. Identification of potential core genes in triple negative breast cancer using bioinformatics analysis. Onco Targets Ther. 2018;11:4105-12.

4. Lv X, He M, Zhao Y, Zhang L, Zhu W, Jiang L, et al. Identification of potential key genes and pathways predicting pathogenesis and prognosis for triple-negative breast cancer. Cancer Cell Int. 2019;19:172. 
5. Wang M, Zhong B, Li M, Wang Y, Yang H, Du K. Identification of potential core genes and pathways predicting pathogenesis in head and neck squamous cell carcinoma. Biosci Rep. 2021; doi:org/10.1042/BSR20204148.

6. Tang Z, Li C, Kang B, Gao G, Li C, Zhang Z. GEPIA: a web server for cancer and normal gene expression profiling and interactive analyses. Nucleic Acids Res. 2017;45(W1):W98-W102.

7. Lamb J, Crawford ED, Peck D, Modell JW, Blat IC, Wrobel MJ, et al. The Connectivity Map: using geneexpression signatures to connect small molecules, genes, and disease. Science. 2006;313(5795):1929-35.

8. Lu L, Zhang J, Gan P, Wu L, Zhang X, Peng C, et al. Novel Functions of CD147 in the Mitochondria Exacerbates Melanoma Metastasis. Int J Biol Sci. 2021;17(1):285-97.

9. Chen J, Peng C, Lei L, Zhang J, Zeng W, Chen X. Nuclear envelope-distributed CD147 interacts with and inhibits the transcriptional function of RING1 and promotes melanoma cell motility. PLoS One.2017; doi:org/10.1371/journal.pone.0183689.

10. Korn EL, Liu PY, Lee SJ, Chapman JA, Niedzwiecki D, Suman VJ, et al. Meta-analysis of phase II cooperative group trials in metastatic stage IV melanoma to determine progression-free and overall survival benchmarks for future phase II trials. J Clin Oncol. 2008;26(4):527-34.

11. Ferlay J, Steliarova-Foucher E, Lortet-Tieulent J, Rosso S, Coebergh JWW, Comber H, et al. Reprint of: Cancer incidence and mortality patterns in Europe: Estimates for 40 countries in 2012. Eur J Cancer. 2015;51(9):1201-2.

12. Thompson JF. Cutaneous melanoma in the era of molecular profiling. Lancet. 2009;374(9687):362-5.

13. Carlino MS, Larkin J, Long GV. Immune checkpoint inhibitors in melanoma. The Lancet. 2021;398(10304):1002-14.

14. Wei SC, Duffy CR, Allison JP. Fundamental Mechanisms of Immune Checkpoint Blockade Therapy. Cancer Discov. 2018;8(9):1069-86.

15. Li H, Kuang X, Liang L, Ye Y, Zhang Y, Li J, et al. The Beneficial Role of Sunitinib in Tumor Immune Surveillance by Regulating Tumor PD-L1. Adv Sci (Weinh). 2021;doi:org/10.1002/advs.202001596.

16. Tran PT, Erdeniz N, Symington LS, Liskay RM. EXO1-A multi-tasking eukaryotic nuclease. DNA Repair (Amst). 2004;3(12):1549-59.

17. JaĖge AC. HNPCC mutations in the human DNA mismatch repair gene hMLH1 in ${ }^{-}$uence assembly of hMutLa and hMLH1 \pm hEX01 complexes. Oncogene. 2001(20)3590 -95.

18. Bolderson E, Tomimatsu N, Richard DJ, Boucher D, Kumar R, Pandita TK, et al. Phosphorylation of Exo1 modulates homologous recombination repair of DNA double-strand breaks. Nucleic Acids Res. 2010;38(6):1821-31.

19. Liberti SE, Andersen SD, Wang J, May A, Miron S, Perderiset M, et al. Bi-directional routing of DNA mismatch repair protein human exonuclease 1 to replication foci and DNA double strand breaks. DNA Repair (Amst). 2011;10(1):73-86. 
20. Tomimatsu N, Mukherjee B, Burma S. Distinct roles of ATR and DNA-PKcs in triggering DNA damage responses in ATM-deficient cells. EMBO Rep. 2009 Jun;10(6):629-35.

21. Schmutte C. Human Exonuclease I Interacts with the Mismatch Repair Protein HMSH2.Cancer Res.1998(58):5437-42.

22. Tomimatsu N, Mukherjee B, Deland K, Kurimasa A, Bolderson E, Khanna KK, et al. Exo1 plays a major role in DNA end resection in humans and influences double-strand break repair and damage signaling decisions. DNA Repair (Amst). 2012;11(4):441-8.

23. Keijzers G, Bakula D, Petr MA, Madsen NGK, Teklu A, Mkrtchyan G, et al. Human Exonuclease 1 (EX01) Regulatory Functions in DNA Replication with Putative Roles in Cancer. Int J Mol Sci.2018; doi.org/10.3390/ijms20010074.

24. Amin NS, Nguyen MN, Oh S, Kolodner RD. exo1-Dependent mutator mutations: model system for studying functional interactions in mismatch repair. Mol Cell Biol. 2001;21(15):5142-55.

25. Genschel J, Kadyrova LY, lyer RR, Dahal BK, Kadyrov FA, Modrich P. Interaction of proliferating cell nuclear antigen with PMS2 is required for MutLalpha activation and function in mismatch repair. Proc Natl Acad Sci U S A. 2017;114(19):4930-5.

26. Qi L, Zhou B, Chen J, Hu W, Bai R, Ye C, et al. Significant prognostic values of differentially expressedaberrantly methylated hub genes in breast cancer. J Cancer. 2019;10(26):6618-34.

27. Yang G, Dong K, Zhang Z, Zhang E, Liang B, Chen X, et al. EXO1 Plays a Carcinogenic Role in Hepatocellular Carcinoma and is related to the regulation of FOXP3. J Cancer. 2020;11(16):4917-32.

28. Dai Y, Tang Z, Yang Z, Zhang L, Deng Q, Zhang X, et al. EXO1 overexpression is associated with poor prognosis of hepatocellular carcinoma patients. Cell Cycle.2018;17(19-20):2386-2397. 29.

29. Luo F, Wang YZ, Lin D, Li J, Yang K. Exonuclease 1 expression is associated with clinical progression, metastasis, and survival prognosis of prostate cancer. J Cell Biochem. 2019. doi:org/10.1002/jcb.28415.

30. Fan J. Phospholipase C- $\varepsilon$ regulates bladder cancer cells via ATM/EXO1. Am J Cancer Res.2020;10(8):2319-2336.

31. Li J, Huang Y, Gao Y, Wu H, Dong W, Liu L. Antibiotic drug rifabutin is effective against lung cancer cells by targeting the elF4E-beta-catenin axis. Biochem Biophys Res Commun. 2016;472(2):299-305.

32. Prati B, da Silva Abjaude W, Termini L, Morale M, Herbster S, Longatto-Filho A, et al. Three Prime Repair Exonuclease 1 (TREX1) expression correlates with cervical cancer cells growth in vitro and disease progression in vivo. Sci Rep. 2019;9(1):351.

33. Liu J, Zhang J. Elevated EXO1 expression is associated with breast carcinogenesis and poor prognosis. Ann Transl Med. 2021;9(2):135.

34. He D, Li T, Sheng M, Yang B. Exonuclease 1 (Exo1) Participates in Mammalian Non-Homologous End Joining and Contributes to Drug Resistance in Ovarian Cancer. Med Sci Monit. 2020; doi:org/10.12659/MSM.918751. 
35. Chen LF, Kaye D. Current use for old antibacterial agents: polymyxins, rifamycins, and aminoglycosides. Infect Dis Clin North Am. 2009;23(4):1053-75.

36. Rothstein DM. Rifamycins, Alone and in Combination. Cold Spring Harb Perspect Med. 2016;doi.org/10.1101/cshperspect.a027011

37. Lee $\mathrm{CY}$, Huang CH, Lu PL, Ko WC, Chen YH, Hsueh PR. Role of rifampin for the treatment of bacterial infections other than mycobacteriosis. J Infect. 2017;75(5):395-408.

38. Kunin CM. Antimicrobial Activity of Rifabutin.1 Clin Infect Dis. 996; doi:org/10.1093/clinids/22.supplement_1.s3.

39. Gisbert JP. Rifabutin for the Treatment of Helicobacter Pylori Infection:A Review.Pathogens.2020; doi:org/10.3390/pathogens 10010015

40. Ribaldone DG, Fagoonee S, Astegiano M, Durazzo M, Morgando A, Sprujevnik T, et al. RifabutinBased Rescue Therapy for Helicobacter pylori Eradication: A Long-Term Prospective Study in a Large Cohort of Difficult-to-Treat Patients. J Clin Med. 2019;8(2).

41. Suzuki $H$, Mori $H$. World trends for $H$. pylori eradication therapy and gastric cancer prevention strategy by H. pylori test-and-treat. J Gastroenterol. 2018;53(3):354-61.

42. Gulla A, Kazlauskas E, Liang H, Strupas K, Petrauskas V, Matulis D, et al. Heat Shock Protein 90 Inhibitor Effects on Pancreatic Cancer Cell Cultures. Pancreas. 2021;50(4):625-32.

43. Elkington1 PT. Implications of tuberculosis reactivation after immune checkpoint inhibition. Am J Respir Crit Care Med.2018. 198(11):1451-1453.

44. Im Y, Lee J, Kim SJ, Koh WJ, Jhun BW, Lee SH. Development of tuberculosis in cancer patients receiving immune checkpoint inhibitors. Respir Med. 2020; doi:org/10.1016/j.rmed.2019.105853

\section{Figures}



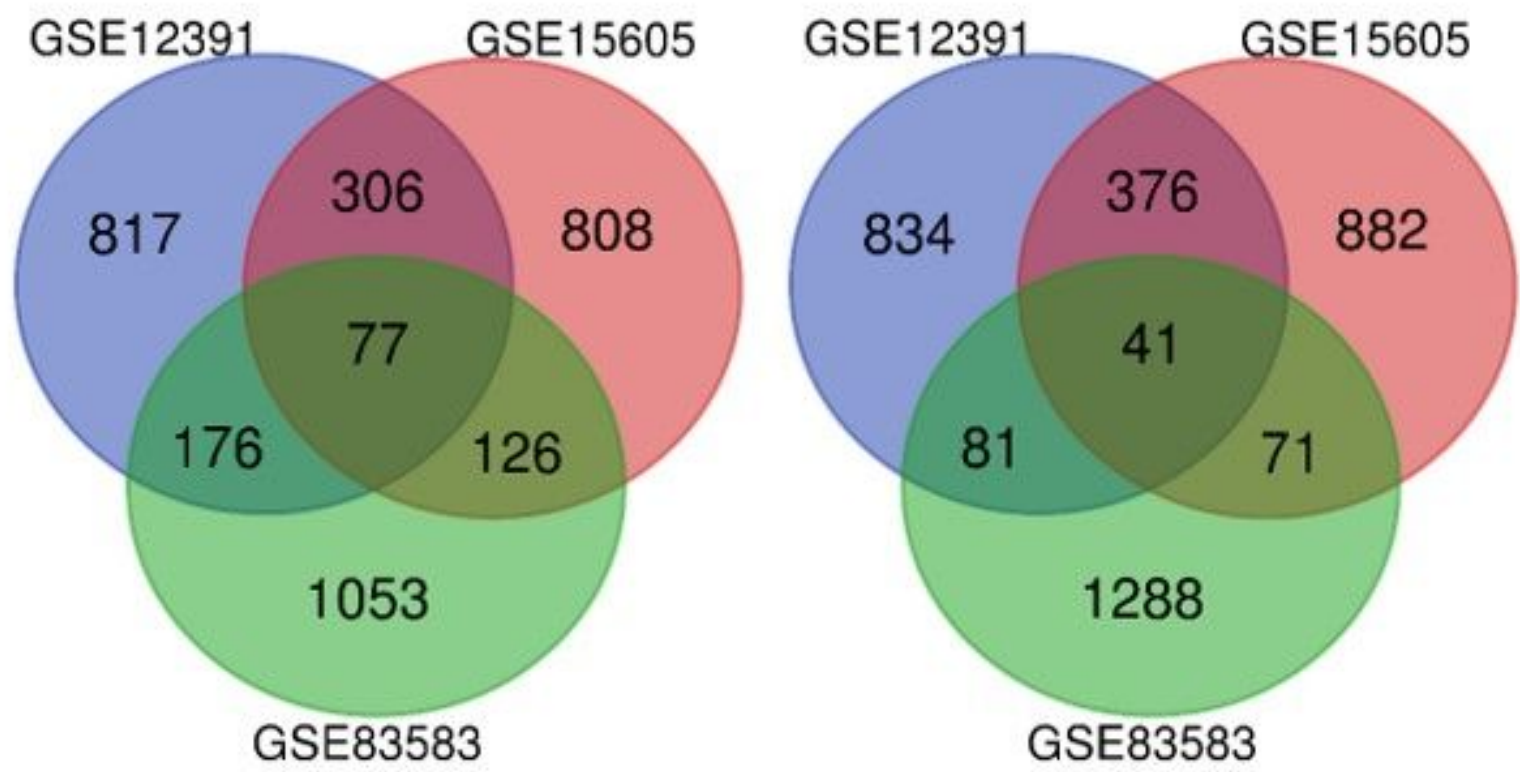

Figure 1

Venn diagram of differentially expressed genes (DEGs) common to all three GEO datasets.

(A) Up-regulated genes; (B) Down-regulated genes.

(A)

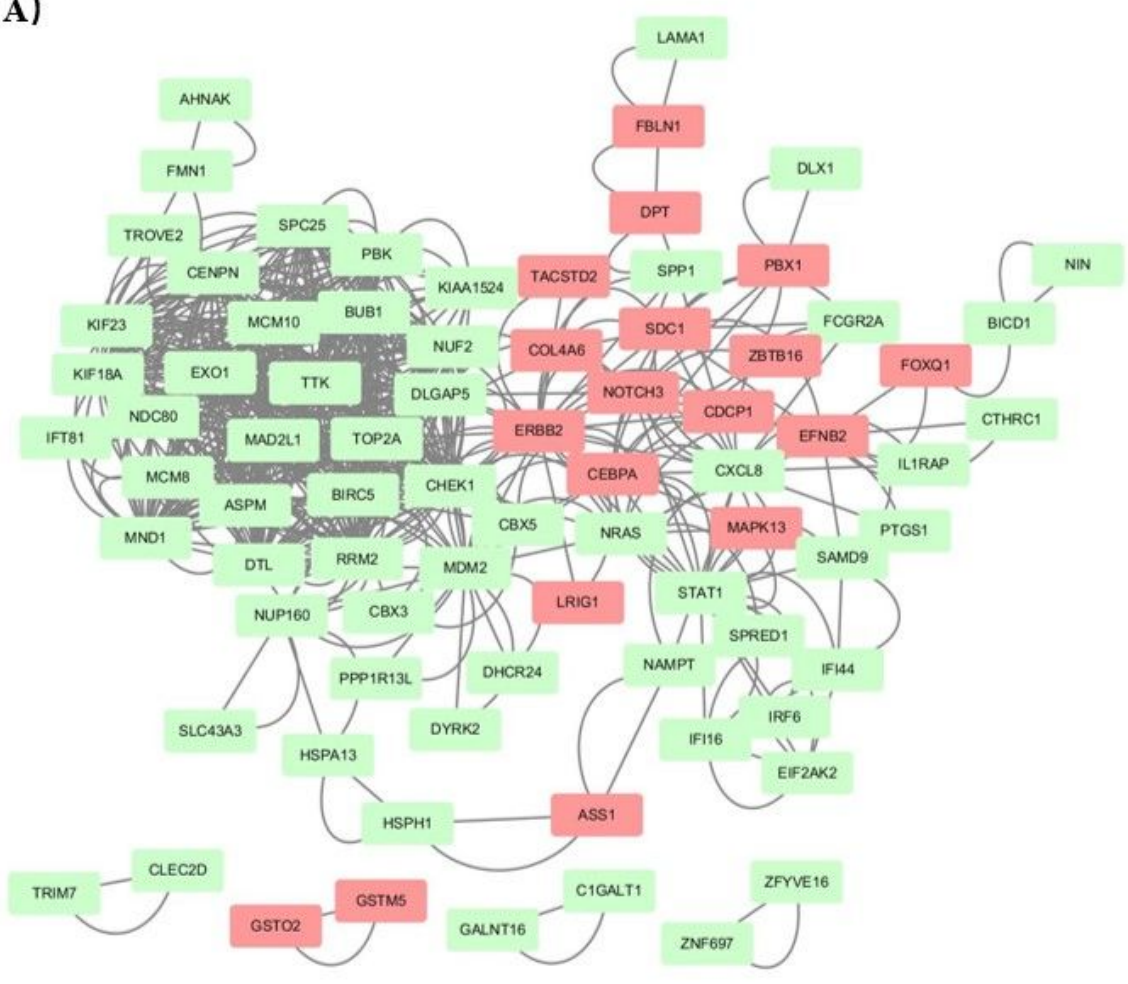

(B)

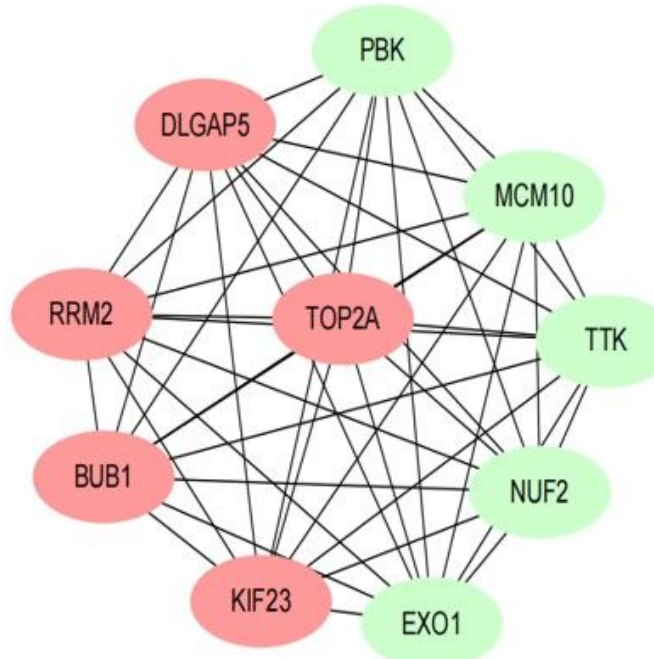

Figure 2 
Protein-protein interaction (PPI) network constructed with the differentially expressed genes (DEGs)

(A) Visualized PPI analysis of DEGs. Green nodes represent up-regulated genes, and red nodes represent down-regulated genes.

(B) The top 10 genes in the PPI network.
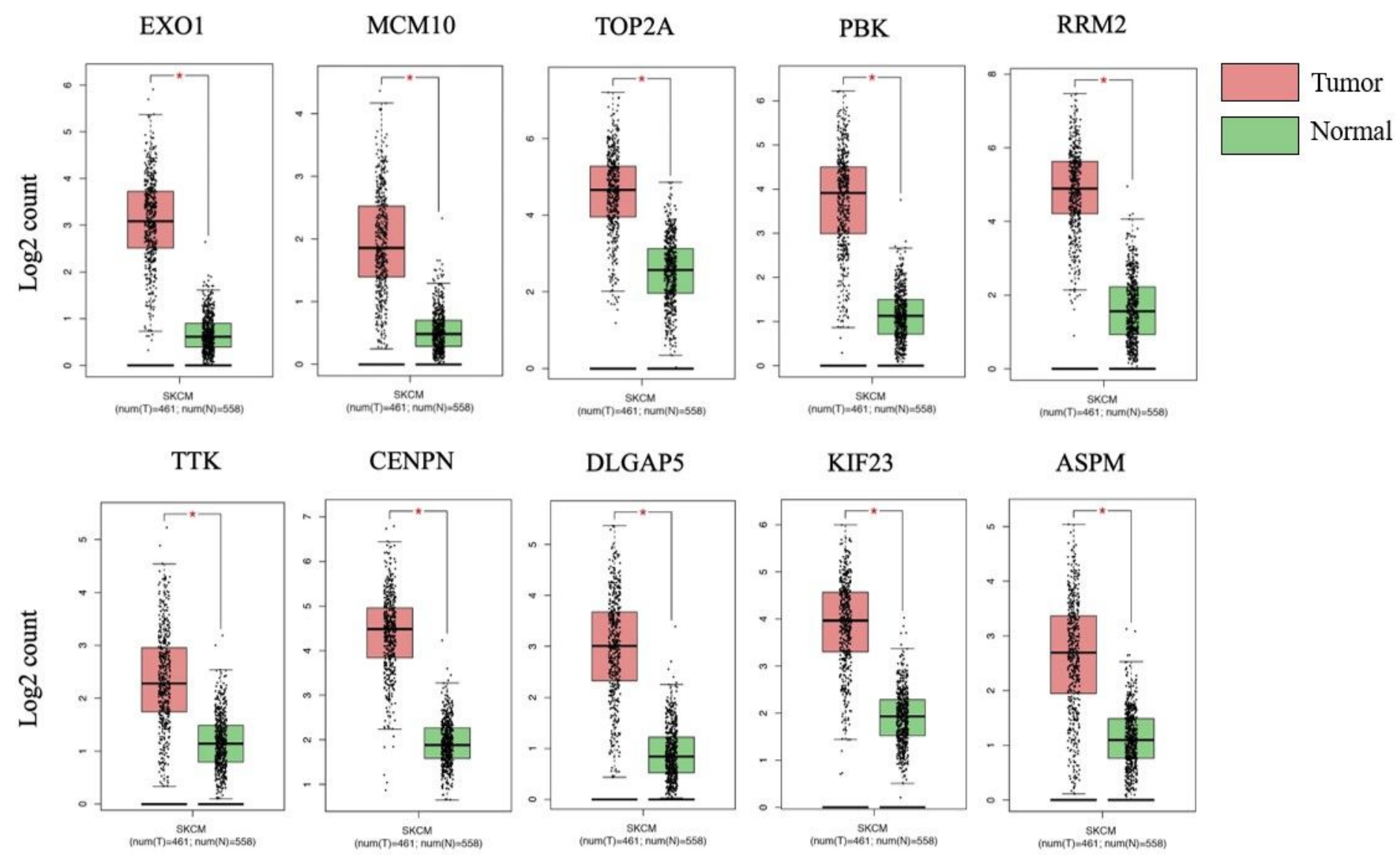

Figure 3

Expression of the ten hub genes in tumor and normal tissues 


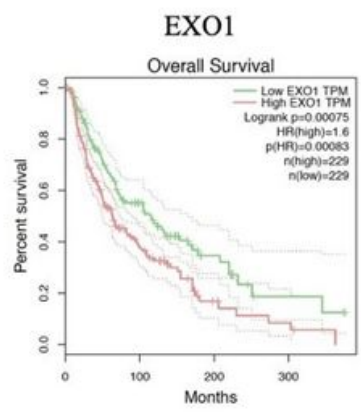

TTK

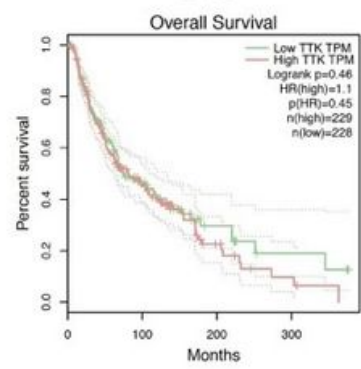

MCM10

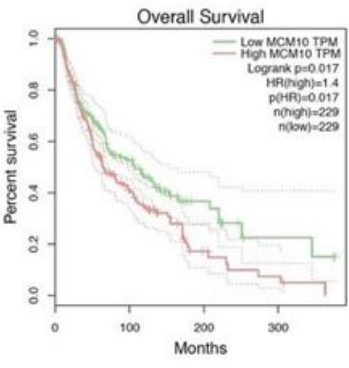

CENPN

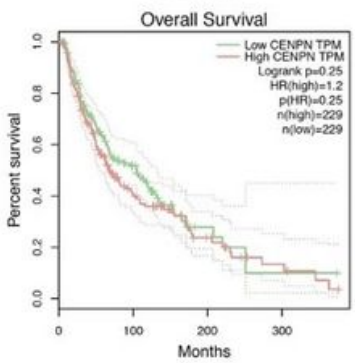

TOP2A

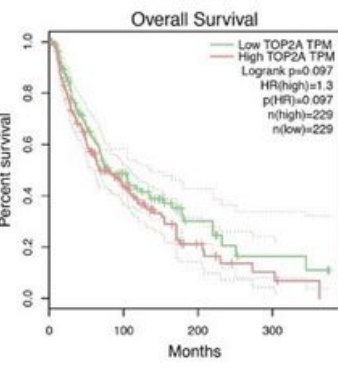

DLGAP5

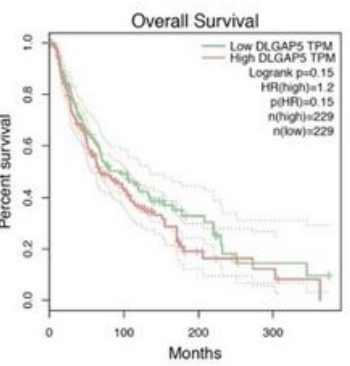

PBK

Overall Survival

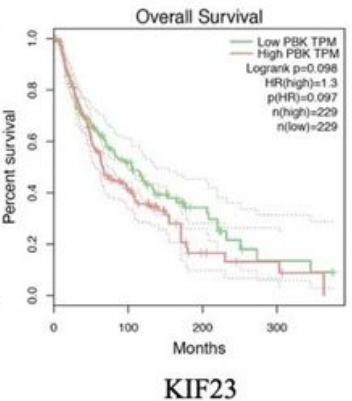

Overall Survival

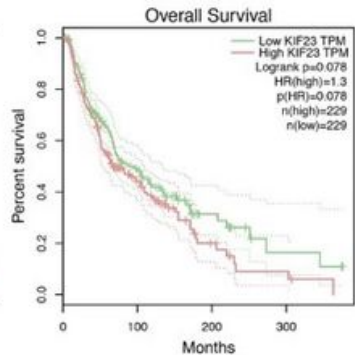

RRM2

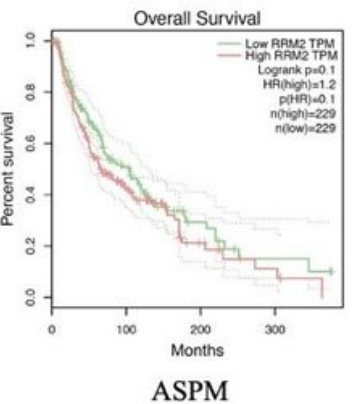

Overall Survival

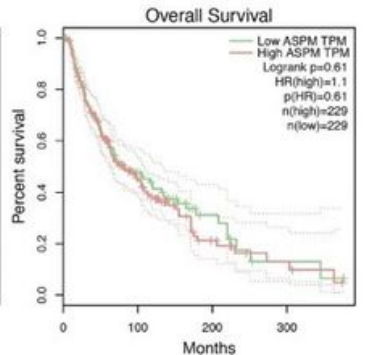

Figure 4

Survival analysis for the top ten hub genes expressed in melanoma patients.

\section{Hacat A375 SK-Mel-28}

EXO1

$\alpha$-Tubulin

\section{Figure 5}

The protein expression of EX01 was analyzed by Western blotting in Hacat, A375 and Sk-Mel-28 cell lines. 


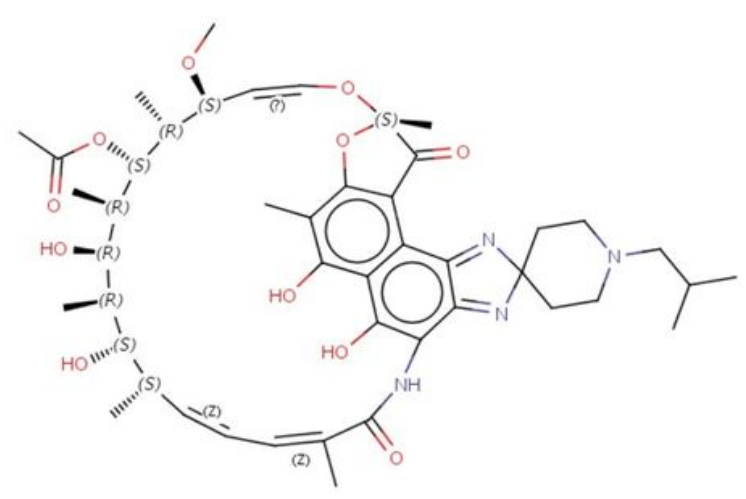

Rifabutin

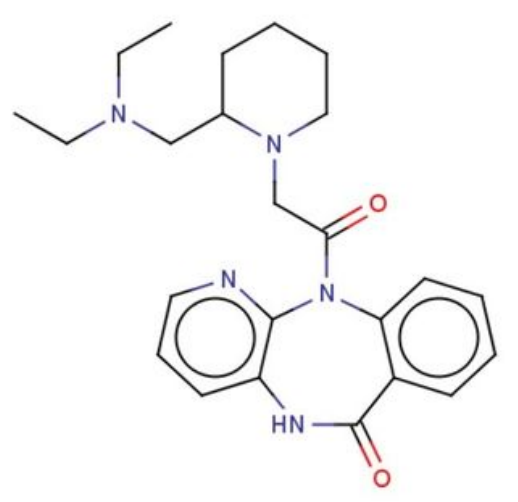

Otenzepad

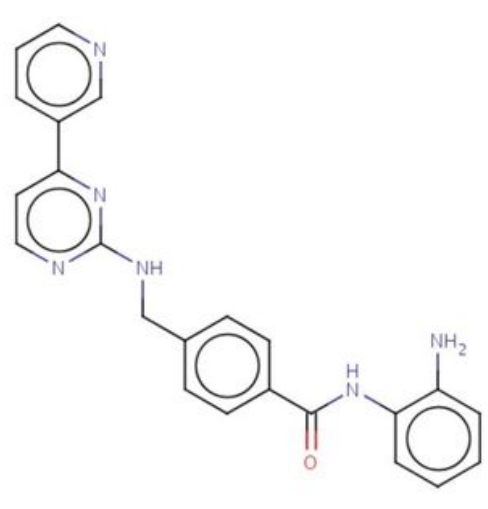

Mocetinostat

Figure 6

Chemical structure of three potential small molecule drugs.

(A)
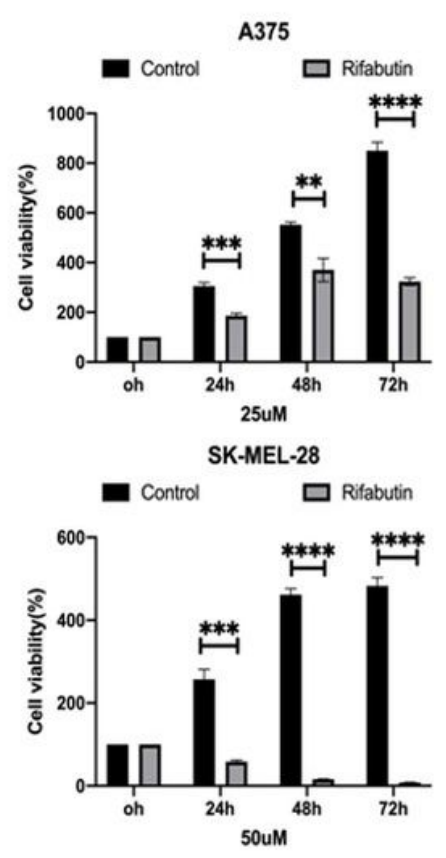

(B)
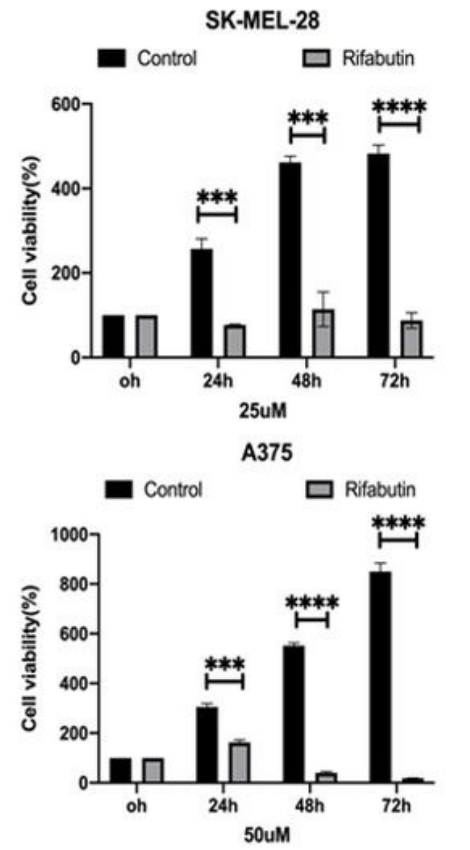

A375

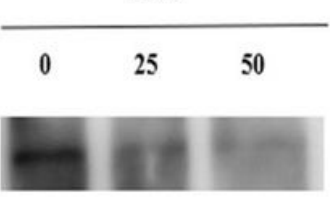

$\alpha$-Tubulin

Rifabutin(um)

EX01

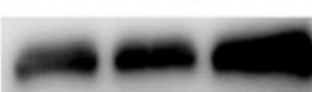

EXO1

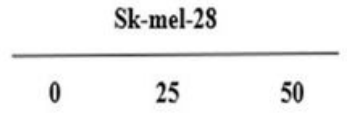

Gapdh

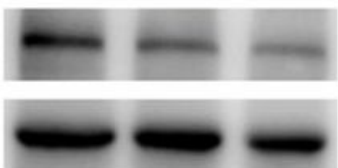

Figure 7

Correlation of the inhibitory effect of rifabutin on melanoma cell proliferation with EXO1 expression.

(A) Estimation of cell viability in A375 and SK-MEL-28 cell lines treated with $25,50 \mu \mathrm{M}$ menadione or distilled water for $0,24,48 \mathrm{~h}, 72 \mathrm{~h}$ by CCK-8 assays. (B) Cells were treated with $0 \mathrm{uM}, 25 \mathrm{uM}, 50 \mu \mathrm{M}$ Rifabutin for $48 \mathrm{~h}$. The expression of EX01 was analyzed by Western blotting; ${ }^{*} P<0.05,{ }^{\star}{ }^{*} \mathrm{P}<0.01$ versus vehicletreated cells. 


\section{Supplementary Files}

This is a list of supplementary files associated with this preprint. Click to download.

- Supplementarymaterial.rar 